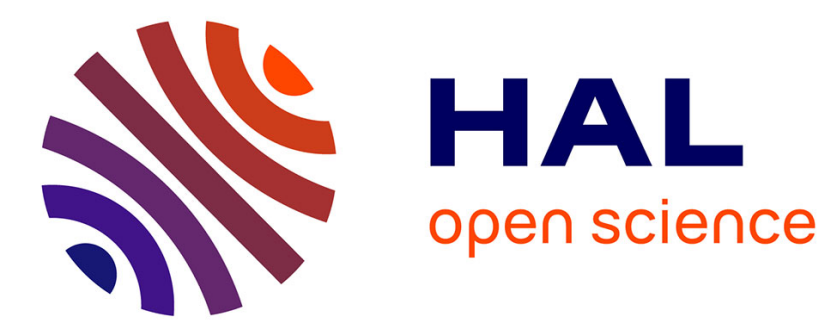

\title{
Un nouvel oscillateur de relaxation
}

Agnès Weill

\section{To cite this version:}

Agnès Weill. Un nouvel oscillateur de relaxation. Revue de Physique Appliquée, 1980, 15 (6), pp.11831191. 10.1051/rphysap:019800015060118300 . jpa-00244840

\section{HAL Id: jpa-00244840 https://hal.science/jpa-00244840}

Submitted on 1 Jan 1980

HAL is a multi-disciplinary open access archive for the deposit and dissemination of scientific research documents, whether they are published or not. The documents may come from teaching and research institutions in France or abroad, or from public or private research centers.
L'archive ouverte pluridisciplinaire HAL, est destinée au dépôt et à la diffusion de documents scientifiques de niveau recherche, publiés ou non, émanant des établissements d'enseignement et de recherche français ou étrangers, des laboratoires publics ou privés. 


\title{
Un nouvel oscillateur de relaxation
}

\author{
A. Weill $\left({ }^{*}\right)$ \\ Ecole d'Application des Hauts Polymères, 4, rue Boussingault, 67000 Strasbourg, France
}

(Reçu le 15 octobre 1979, révisé le 19 février 1980, accepté le 22 février 1980)

\begin{abstract}
Résumé. - En forçant l'écoulement d'un polyéthylène de haute densité fondu à travers une filière capillaire, on crée, sous certaines conditions, un régime vibratoire. La pression et le débit sont des fonctions périodiques du temps. Nous en concluons que l'instabilité d'écoulement des polymères fondus doit s'analyser en terme d'oscillations de relaxation. Il est alors possible de réduire la dépendance du système physique aux deux paramètres pertinents que sont la résistance et la capacité.
\end{abstract}

\begin{abstract}
The flow of high density polyethylene melt can give, under certain conditions, oscillatory working conditions. Pressure and flow rate are periodical functions of time. It can be concluded that instable flow of polymer melts must be regarded as relaxation oscillators. So, the variations of this mechanical system can be reduce to the variations of the two pertinent parameters, i.e. the resistance and the capacity functions.
\end{abstract}

Introduction. - La physique des mouvements vibratoires peut se diviser en deux : le domaine des oscillations harmoniques et celui des oscillations à caractère relaxatif. Les deux familles d'oscillations ont des exemples représentatifs dans des branches scientifiques aussi diverses que l'électricité, l'acoustique, la chimie, etc...

Nous avons récemment mené à bien à l'Ecole d'Application des Hauts Polymères une recherche portant sur la stabilité des écoulements de polymères fondus [1]. D'autres travaux certes avaient été réalisés depuis longtemps [2, 3, 4] cherchant à préciser aussi bien la nature que l'origine des instabilités d'écoulement. Celles-ci apparaissent lorsque la contrainte de cisaillement à la paroi de la filière atteint une valeur critique ( $\simeq 1$ à 4 bar). Les travaux précédents $[2,3,4]$ ont montré que les instabilités se manifestent par l'apparition d'écoulements périodiques de grande amplitude. Mais aucun d'entre eux ne s'est attaché à décrire et expliquer l'évolution de la période d'oscillation en fonction des conditions expérimentales.

L'objectif est donc de proposer une théorie phénoménologique qui permette de mettre en évidence les paramètres pertinents qui règlent la période d'oscillation.

En nous restreignant au cas du polyéthylène de haute densité, nous avons proposé une analyse du

$\left(^{*}\right)$ Nouvelle adresse : C.N.E.T., 4, chemin des Prés, Locazirst, 38240 Meylan, France. phénomène en terme d'oscillations de relaxation qui s'est révélée fructueuse. L'objet de cet article est de présenter cette analyse et d'identifier les oscillations observées.

Dans la première partie, nous nous attachons à décrire le phénomène. La deuxième est consacrée à l'analyse quantitative.

1. Présentation phénoménologique: - 1.1 APPAREILlAGE ET MATIÈRE. - L'appareil utilisé est un rhéomètre capillaire (Fig. 1) du type ZWICK no 7901. La matière est initialement contenue dans un canal d'essai dans lequel coulisse un piston. Celui-ci est relié à la traverse d'une machine d'essais mécaniques par l'intermédiaire d'une bille de guidage et d'une cellule de mesure de force. Afin d'éviter les fuites, le canal d'essai est fortement appliqué contre la filière. La température du canal et de la filière est constante à un demi-degré près.

Lorsque la traverse est mise en mouvement, elle crée de part et d'autre de la filière une différence de pression et le fluide s'écoule.

Selon la conception de l'appareil, la vitesse de la traverse est une grandeur indépendante, alors que la force exercée sur le piston est une grandeur dépendante. La valeur de la vitesse de la traverse est, en effet, issue du choix de l'expérimentateur et ne dépend en aucune manière des conditions d'expérience : matière utilisée, température, géométrie du système physique, etc... Par contre l'intensité de la force exercée sur le piston par la matière est $a$ priori inconnue de l'expéri- 


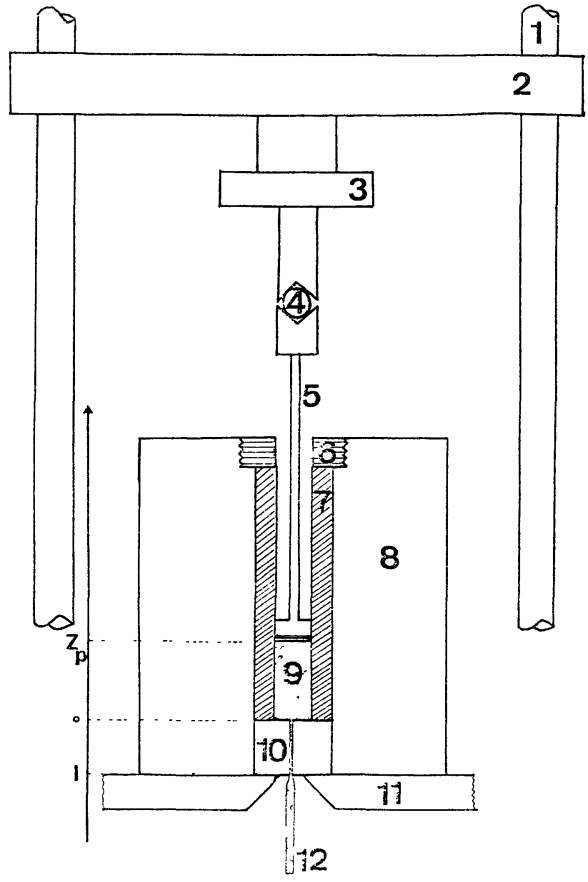

Fig. 1. - Le rhéomètre capillaire : 1) colonnes de la machine d'essais, 2) traverse, 3) cellule de mesure de force, 4) bille de guidage, 5) piston, 6) vis de serrage, 7) canal d'essai, 8) four, 9) réservoir, 10) filière, 11) support, 12) extrudat.

[Capillary rheometer : 1) drive system, 2) crosshead, 3) stress gauge transducer, 4) ball, 5) piston, 6) screw, 7) barrel, 8) oven, 9) reservoir, 10) die, 11) bearer, 12) extrudate.]

mentateur et complètement déterminee par les conditions d'expérience.

Dans un souci de simplification, les résultats exposés ici ne concernent qu'un seul polyéthylène de haute densité à $190^{\circ} \mathrm{C}$, c'est-à-dire bien au-delà du point de fusion $\left(134^{\circ} \mathrm{C}\right)$. Ses caractéristiques moléculaires sont :

- Masse moyenne en poids : $130000 \mathrm{~g} / \mathrm{mol}$.

- Masse moyenne en nombre : $24000 \mathrm{~g} / \mathrm{mol}$.

Il s'agit d'un polyéthylène type industriel (extrusion soufflage). Mais il convient de noter que tous les polyéthylènes de haute densité de haut poids moléculaire sont le siège de phénomènes identiques dans une gamme de température très large.

1.2 Système PHysique. - 1.2.1 Définition. - Le système physique est défini par l'espace compris entre la sortie de la filière et le piston. Le volume de ce système varie linéairement au cours du temps lorsque la traverse de la machine d'essai est mise en action.

1.2.2 Approximation quasi statique. - Nous supposons dans tout l'exposé qui suit que les durées d'observation (ou de mesure) sont suffisamment grandes et que les dimensions du système physique étudié sont suffisamment petites pour que le phénomène de propagation d'onde de pression dans le système soit d'influence négligeable. Cela signifie en particulier qu'à chaque instant la valeur de la pression est la même en tout point d'un liquide immobile. Grâce à cette hypothèse, la pression à l'entrée de la filière peut être confondue avec celle qui règne au voisinage du piston. En fixant à $20 \mathrm{~cm}$ les dimensions maxima du réservoir et en évaluant la vitesse de propagation des ondes de dilatation volumique à $1000 \mathrm{~m} / \mathrm{s}$, nous voyons que des phénomènes périodiques de fréquence $v$ ne sont pas perturbés par la propagation d'ondes de dilatation volumiques quand $v$ est petit devant $v_{\mathrm{s}}$

$$
v \ll v_{\mathrm{s}}=\frac{1000 \mathrm{~m} / \mathrm{s}}{0,2 \mathrm{~m}}=5000 \mathrm{~Hz} .
$$

1.2.3 Grandeurs mécaniques. - Quatre grandeurs mécaniques caractérisent macroscopiquement le système physique.

- Le produit de la vitesse $V_{\mathrm{p}}$ du piston par la surface $S_{\mathrm{c}}$ du canal. C'est une constante dans le temps appelée débit volumique à l'entrée du système, ou plus simplement débit d'entrée

$$
Q_{\mathrm{E}}=V_{\mathrm{p}} \times S_{\mathrm{c}} .
$$

- Le rapport de la force $F_{\mathrm{p}}$ exercée par la matière sur le piston à la surface $S_{\mathrm{c}}$ de la section du canal d'essai. C'est une fonction du temps que nous appelons par définition pression à l'entrée du système, ou pression d'entrée

$$
P_{\mathrm{E}}(t)=F_{\mathrm{p}} / S_{\mathrm{c}} .
$$

- Le débit volumique de matière à la sortie du système, ou débit de sortie est une fonction du temps

$$
Q_{\mathrm{s}}(t) \text {. }
$$

- La pression à la sortie du système, égale à celle du milieu extérieur, est une constante dans le temps

$$
P_{\mathrm{S}}=P_{\text {atmosphérique }} \text {. }
$$

Le système physique est caractérisé par l'évolution dans le temps d'une grandeur extensive $Q(t, z)$ et d'une grandeur intensive $P(t, z)$. L'abscisse $z$ mesure les distances le long de l'axe de symétrie du montage.

Les conditions aux limites sont :

$$
\begin{aligned}
& P(t, 0)=P_{\mathrm{E}}(t) \\
& P(t, 1)=P_{\mathrm{atm}} \\
& Q(t, 1)=Q_{\mathrm{S}}(t) \\
& Q(0,1)=0 .
\end{aligned}
$$

Les extrémités de la filière étant situées aux points d'abscisse $z=0$ et $z=1$.

1.2.4 Conditions d'expérience. - Elles sont définies par :

- La matière : Nous intégrons dans ce paramètre la structure physique et la nature chimique de la chaîne macromoléculaire. 
- La température : Elle est supposée uniforme dans tout le système.

- La géométrie du montage : Il s'agit des diamètres du canal d'essai et de la filière, la longueur de la filière, l'angle du cône d'entrée de la filière.

Il va de soi que les grandeurs que nous venons de citer comme paramètres ne varient pas durant tout le déroulement d'une expérience. Nous supposons en particulier que le polymère ne subit aucune dégradation thermique ou mécanique, que la température du système n'évolue pas dans le temps et que les variations de pression subies par le montage n'affectent pas sensiblement ses dimensions géométriques.

1.2.5 Les résultats d'expérience. - Dans le cadre de cet article, nous nous bornerons à étudier l'évolution des grandeurs mécaniques avec le temps : $P_{\mathrm{E}}(t)$ et $Q_{\mathrm{S}}(t)$.

1.3 DESCRIPTION TYPE D'UN COMPORTEMENT. Afin de faciliter la description nous allons de manière arbitraire fixer les valeurs des paramètres expérimentaux suivants :

température : $190^{\circ}$,

canal : $\varnothing 16 \mathrm{~mm}$,

filière : entrée plate $l=10 \mathrm{~mm} ; \varnothing=0,5 \mathrm{~mm}$.

Les courbes représentant les fonctions $Q_{\mathrm{s}}(t)$ et $P_{\mathrm{E}}(t)$ montrent qu'il existe quatre régimes de fonctionnement selon la valeur du débit d'entrée (Fig. 2). Bien que Lupton et Register [5] aient déjà partiellement décrit ce fonctionnement, nous reprenons cette description pour la compléter et pour en faire une interprétation dynamique.
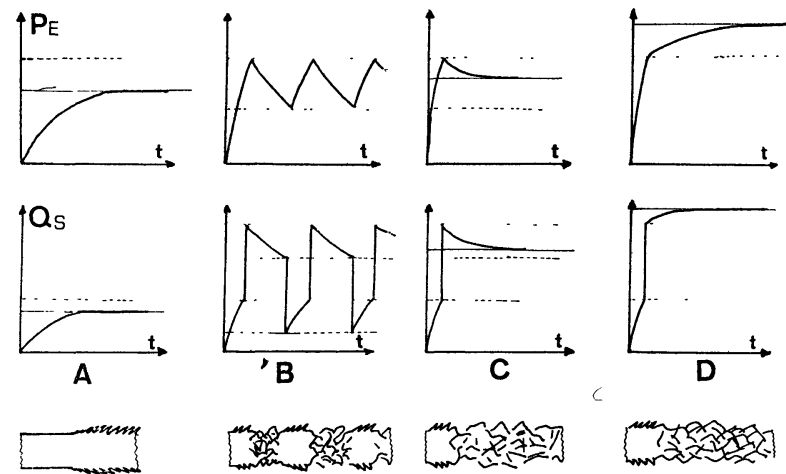

Fig. 2. - Evolution de la pression d'entrée $P_{\mathrm{E}}$ et du débit de sortie $Q_{\text {s }}$ en fonction du temps et représentation schématique de la forme de l'extrudat pour les quatre régimes : A) stable, B) oscillatoire, C) pic de pression, D) stable avec discontinuité.

[The variation of the entry pressure, $P_{\mathrm{E}}$, and the exit flow rate, $Q_{\mathrm{s}}$, with the time, $t$, and schematical aspect of the extrudate for the four working conditions : A) stable, B) oscillatory; C) pressure peak, D) stable with discontinuity.]

Bergem [6] a aussi observé un changement dans la forme des graphes $Q_{\mathrm{E}}(t)$ mais sans les interpréter.

Le débit d'entrée est supposé passer instantanément d'une valeur nulle à une valeur finie positive $Q_{\mathrm{E}}$ au temps $t=0$.
Les fonctions $Q_{\mathrm{s}}(t)$ et $P_{\mathrm{E}}(t)$ sont schématisées dans la figure 2 pour chacun des quatre régimes de fonctionnement. Elles sont toutes les deux initialement nulles

$$
P_{\mathrm{E}}(t=0)=Q_{\mathrm{S}}(t=0)=0 .
$$

1.3.1 Régime stable $Q_{\mathrm{E}}<5,6 \mathrm{~mm}^{3} / \mathrm{s}$. - Le graphique indique que les fonctions $P_{\mathrm{E}}(t)$ et $Q_{\mathrm{S}}(t)$ sont croissantes et monotones. Quand $t$ tend vers l'infini elles tendent vers une valeur finie notée respectivement $P_{\text {eq }}$ et $Q_{\text {eq }}$.

Une simple analyse du bilan massique montre que, pour un temps infini,

$$
Q_{\mathrm{eq}}=Q_{\mathrm{E}}=Q_{\mathrm{S}}
$$

le débit de sortie $Q_{\mathrm{S}}$ est égal au débit d'entrée $Q_{\mathrm{E}}$. Les valeurs $Q_{\text {eq }}$ et $P_{\text {eq }}$ peuvent être utilisées pour calculer la viscosité apparente en cisaillement simple du fluide (écoulement permanent).

Le régime transitoire initial a une durée de vie de quelques dizaines de secondes. Il est dû à la valeur non nulle de la compressibilité du polymère fondu. Il existe un déphasage, ou un retard, entre la fonction signal $Q_{\mathrm{E}}$ et la fonction réponse $Q_{\mathrm{S}}(t)$.

Si la compressibilité $\chi$ était nulle il n'y aurait aucun retard et si elle était infinie il n'y aurait aucun écoulement

$$
\begin{array}{ll}
\chi=0 \rightarrow \forall t>0 & Q_{\mathrm{s}}(t)=Q_{\mathrm{E}} \\
\chi=\infty \rightarrow \forall t>0 & Q_{\mathrm{s}}(t)=0 .
\end{array}
$$

L'étude de la forme de la fonction $P_{\mathrm{E}}(t)$ au cours du régime transitoire permet de calculer la compressibilité hydrostatique du fluide.

Pour les faibles valeurs de $P_{\mathrm{E}}(t)$ la surface de l'extrudat est lisse et régulière. Par contre quand $P_{\mathrm{E}}(t)$ at teint la valeur $P_{\mathrm{E}}=2,2 \times 10^{7} \mathrm{~Pa}$ l'extrudat présente une certaine rugosité de surface décrite dans la littérature sous le nom de peau de requin (sharkskin). Dans une publication antérieure [7] nous donnons notre point de vue quant à l'origine de ce phénomène.

1.3.2 Régime oscillatoire $5,6<Q_{\mathrm{E}}<25 \mathrm{~mm}^{3} / \mathrm{s}$. - Le régime transitoire initial est identique au cas précédent. Mais les fonctions $P_{\mathrm{E}}(t)$ et $Q_{\mathrm{S}}(t)$ n'atteignent pas de valeur d'équilibre pour $t$ grand. Elles oscillent entre deux valeurs extrêmes constantes dans le temps. Comme l'indique la figure 2, ce sont des fonctions périodiques du temps. Elles ont même période : quelques secondes environ. L'étude et la caractérisation des oscillations de pression et de débit est l'objectif du présent article. Nous étudierons principalement l'influence des paramètres d'expérience sur la fréquence des oscillations. Notons seulement ici que le débit d'entrée n'influence pas l'amplitude des oscillations mais joue sur la période.

La loi de la conservation de la matière impose que 
la quantité débitée pendant une période soit égale au produit de la période par le débit d'entrée

$$
\frac{1}{T} \int_{T} Q_{\mathrm{S}}(t) \mathrm{d} t=Q_{\mathrm{E}}
$$

La forme du jonc extrudé (voir Fig. 2) est aussi une fonction périodique de la longueur. $\mathrm{La}$ période spatiale correspond à la période temporelle des fonctions $P_{\mathrm{E}}(t)$ et $Q_{\mathrm{S}}(t)$. Pendant l'intervalle de temps durant lequel la pression est croissante, $\mathrm{d} P_{\mathrm{E}} / \mathrm{d} t>0$, le jonc a exactement le même aspect que dans le cas du régime stable décrit ci-dessus. A l'instant précis où brusquement la pression devient décroissante, $\mathrm{d} P_{\mathrm{E}} / \mathrm{d} t<0$, une sorte de bouchon est violemment expulsé de la filière. Tout se passe apparemment comme si ce bouchon retenait en partie le fluide. En effet, après l'expulsion, le débit de sortie $Q_{\mathbf{s}}(t)$ prend des valeurs extrêmement élevées et la pression décroît. L'extrudat n'a plus de forme définie et son profil paraît être purement aléatoire. La partie de l'extrudat qui correspond à la décroissance de $P_{\mathrm{E}}(t)$ sera dite chaotique, par opposition à la zone relativement bien organisée qui présente des défauts du type peau de requin.

1.3.3 Régime de pic de pression $25<Q_{\mathrm{E}}<85 \mathrm{~mm}^{3} / \mathrm{s}$. - Les fonctions $P_{\mathrm{E}}(t)$ et $Q_{\mathrm{S}}(t)$ ont une allure identique aux fonctions décrites dans le cas précédent, durant la première oscillation. Mais durant la décroissance de pression, $P_{\mathrm{E}}(t)$ et $Q_{\mathrm{S}}(t)$ tendent vers une valeur d'équilibre $P_{\text {eq }}$ et $Q_{\text {eq' }}$ comme si la période était devenue infiniment grande

$$
\begin{aligned}
t \rightarrow \infty \frac{\mathrm{d} P_{\mathrm{E}}}{\mathrm{d} t} \rightarrow 0^{-} \quad P_{\mathrm{E}} \rightarrow P_{\mathrm{eq}}, \\
\frac{\mathrm{d} Q_{\mathrm{S}}}{\mathrm{d} t} \rightarrow 0^{-} \quad Q_{\mathrm{S}} \rightarrow Q_{\mathrm{eq}} .
\end{aligned}
$$

Pour les mêmes raisons que plus haut la valeur d'équilibre du débit de sortie est égale au débit d'en trée. Après la partie caractéristique de la montée en pression et de l'expulsion du bouchon, le jonc extrudé est indéfiniment chaotique.

1.3.4 Régime stable avec une discontinuité $Q_{\mathrm{E}}>85 \mathrm{~mm}^{3} / \mathrm{s}$. - Pour les débits d'entrée extrêmement élevés la description du comportement du fluide est identique au cas précédent jusqu'à l'instant d'expulsion du bouchon. Dans le cas présent il n'y a pas de décroissance de pression. Le système tend vers un état d'équilibre mécanique, la courbe $Q_{\mathrm{s}}(t)$ présente une seule discontinuité et la courbe $P_{\mathrm{E}}(t)$ une seule discontinuité de pente

$$
\forall t, \quad \frac{\mathrm{d} P_{\mathrm{E}}}{\mathrm{d} t}>0
$$

Ici aussi, le débit de sortie tend vers une valeur d'équilibre égale au débit d'entrée. Le jonc extrudé a le

même aspect que lors du régime à pic de pression. L'écoulement n'étant pas laminaire, on ne peut pas calculer la viscosité.

1.3.5 Diagramme Pression-Débit (Fig. 3). - En éliminant le paramètre $t$ entre les deux fonctions $P_{\mathrm{E}}(t)$ et $Q_{\mathrm{S}}(t)$ nous arrivons à une représentation du fonctionnement du système dans un diagramme pression d'entrée-débit de sortie. La courbe obtenue matérialise la trajectoire d'un point mobile $\mathrm{F}$, appelé point de fonctionnement.
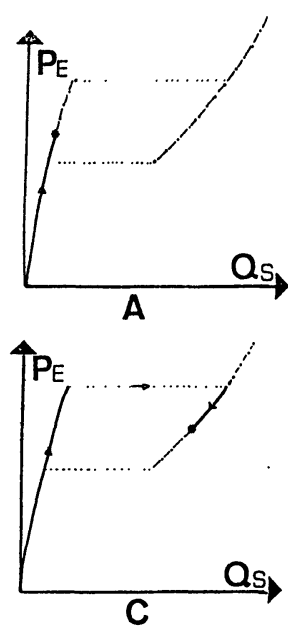

Fig. 3. - Diagramme pression-débit obtenu à partir des courbes de la figure 2 par élimination du paramètre $t$.

[Pressure-flow diagram as obtained from the curves of the figure 2 by eliminating the parameter $t$.]

A chaque instant, l'état du système est représenté par un point $F$ situé sur l'une des courbes de la figure 3 . Il n'y a que dans le régime 2 (Fig. $3 b$ ) que la vitesse du point $F$ n'est pas nulle pour un temps suffisamment long. Dans ce cas le point décrit indéfiniment un cycle dans le sens indiqué par les flèches.

1.4 INTERPRÉTATION DYNAMIQUE DU COMPORTEMENT. - 1.4.1 Courbe d'écoulement non monotone.A partir d'une théorie thermodynamique de l'écoulement d'un fluide viscoélastique et en mesurant le spectre de relaxation d'un polyéthylène de haute densité, Huseby [16] a montré que ce polymère présente une relation contrainte-vitesse de cisaillement $s(\dot{\gamma})$ non monotone, schématisée dans la figure 4 .

D'autre part, on sait que, quelle que soit la nature du fluide, la contrainte de cisaillement en un point $\mathbf{M}$ d'un fluide en écoulement dans un capillaire, est directement proportionnelle à la coordonnée radiale de $M$ et au gradient axial de pression en ce point

$$
s=\frac{r}{2} \frac{\partial P}{\partial z} .
$$

Quelle est la conséquence de la non-monotonie de $s(\dot{\gamma})$ sur le diagramme $P(Q)$ correspondant?

Si la contrainte de cisaillement à la paroi, $s_{\mathrm{p}}$, est 


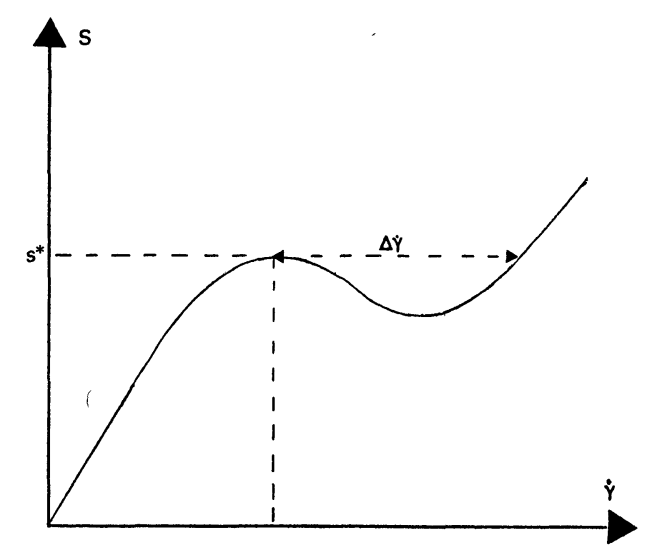

Fig. 4. - Courbe d'écoulement non monotone contrainte-vitesse de cisaillement donnant naissance à un écoulement instable.

[Non monotoneous shear stress-shear rate curve, giving rise to instable flow.]

inférieure à la valeur critique $s^{*}$ correspondant au point de dérivée nulle,

$$
\left.\frac{\mathrm{d} s}{\mathrm{~d} \gamma}\right]_{s=s^{*}}=0
$$

il est clair qu'une augmentation régulière du gradient de pression induit une augmentation continue du débit volumique.

Mais si la contrainte de cisaillement $s_{\mathrm{p}}$ atteint la valeur critique $s^{*}$, le profil radial de vitesse de cisaillement $\dot{\gamma}(r)$ à l'intérieur du tube se trouve alors être une fonction discontinue. Cet échelon de vitesse de cisaillement $\Delta \dot{\gamma}$ induit une augmentation brutale du débit $\Delta Q$, représentée dans la figure $3 b$ par le trait pointillé $(\mathrm{a}-\mathrm{b})$.

Or le point (b) ne peut être un point d'équilibre car dans ces conditions le débit de sortie $Q_{\mathrm{s}}$ se trouve être supérieur au débit d'entrée $Q_{\mathrm{E}}$. Il suit alors une période de décompression représentée dans la figure $3 b$ par le chemin (b-c). Et si le débit de sortie correspondant au point (c) est encore supérieur au débit d'entrée, un deuxième saut de débit a lieu, selon le chemin (c-d). Au point (d), le débit de sortie est devenu inférieur au débit d'entrée, le régime de compression s'établit à nouveau et un second cycle (abcd) prend naissance.

Les temps de commutation (ab) et (cd) sont extrêmement brefs (environ $10^{-2} \mathrm{~s}$ ) devant la période nécessaire à l'accomplissement d'un cycle entier (environ 1 à $100 \mathrm{~s}$ ).

Nous nous proposons dans la partie qui suit de calculer les périodes d'oscillation. Les temps de commutation étant négligeables, la période d'oscillation se décompose en une partie de compression et une partie de décompression.

1.4.2 Elasticité du polymère. - La compressibilité du polymère stocké dans le réservoir joue un grand rôle dans les phénomènes transitoires et oscillatoires que nous venons de décrire. C'est parce que le polymère fondu est compressible que le débit de sortie
$Q_{\mathrm{S}}(t)$ peut ne pas être égal à chaque instant à la valeur du débit d'entrée. C'est bien le caractère élastique du polymère qui est ici en jeu et non pas son caractère visqueux.

Le même phénomène de retard observé lors du premier régime stable existe pour un liquide newtonien ordinaire tel que l'eau ou l'alcool, eux aussi compressibles. La seule différence, de ce point de vue, entre un fluide polymère et un fluide moléculaire est la durée du régime transitoire.

La compressibilité du polymère est sûrement une condition nécessaire à son existence, puisque lorsque ce régime est établi le débit de sortie n'est jamais égal au débit d'entrée.

1.4.3 Durée d'un régime transitoire. - A l'aide du schéma représenté dans la figure 1, dressons un bilan d'échange de matière entre le système physique et le milieu extérieur : pendant un instant infiniment bref $" \mathrm{~d} t$ " le piston avance d'une longueur infiniment petite $" \mathrm{~d} z »$. La masse de matière qui s'échappe du système par la filière est notée $" \mathrm{~d} m$ ».

Soit $S_{\mathrm{c}}$ la section du canal d'essai, $z(t)$ la distance du piston à la filière, $\rho(t)$ la masse volumique. Si le débit de sortie à l'instant $t$ est $Q_{\mathrm{s}}, \mathrm{d} m$ s'écrit selon (1).

$$
\mathrm{d} m(t)=\rho(t) Q_{\mathrm{S}}(t) \mathrm{d} t .
$$

La variation de masse contenue dans le réservoir est égale à la variation du produit du volume du réservoir par sa masse volumique. Puisqu'il n'y a pas de fuite il vient :

$$
\rho Q_{\mathrm{s}} \mathrm{d} t+\mathrm{d}\left(S_{\mathrm{c}} \rho z\right)=0 .
$$

Nous voulons évaluer comparativement l'ordre de grandeur de la durée de vie d'un régime transitoire entre un fluide moléculaire et un fluide polymère. Nous choisissons donc le régime transitoire le plus simple à analyser mathématiquement : c'est celui qui suit un régime permanent lorsque le débit d'entrée passe instantanément d'une valeur $Q_{\mathrm{E}}$ à une valeur nulle $(\mathrm{d} z / \mathrm{d} t=0$ pour $t>0)$. L'analyse se simplifie encore en supposant les fluides newtoniens, c'est-àdire en supposant une relation (3) linéaire entre le débit et la pression. Nous cherchons, dans ces conditions, à calculer l'intervalle de temps noté $t_{99}$ nécessaire pour que la pression décroisse de $99 \%$ de sa valeur initiale.

$$
Q_{\mathrm{S}}(t)=\frac{\alpha}{\eta_{0}} P_{\mathrm{E}}(t)
$$

$\alpha$ : constante dépendant des caractéristiques géométriques du montage, $\eta_{0}$ : viscosité du fluide.

La compressibilité $\chi$ d'un fluide est définie en thermodynamique par la relation (4). La relation linéaire entre le volume et la densité (5) permet de transformer (2) en (6), puis en (7). 


$$
\begin{gathered}
\chi=-\frac{1}{v} \frac{\mathrm{d} v}{\mathrm{~d} P} \\
{\left[\frac{\mathrm{d} v}{v}\right]_{m}=-\frac{\mathrm{d} \rho}{\rho}} \\
\frac{1}{\eta_{\mathrm{O}}} \alpha P_{\mathrm{E}}(t) \mathrm{d} t+S_{\mathrm{c}} z \chi \mathrm{d} P_{\mathrm{E}}=0 \\
\mathrm{~d} t=\frac{S_{\mathrm{c}}}{\alpha} \eta_{0} \chi z \frac{\mathrm{d} P_{\mathrm{E}}}{P_{\mathrm{E}}} .
\end{gathered}
$$

L'intégration de l'équation (7) est une exponentielle. Il en découle (8) :

$$
t_{99}=4,6 S_{\mathrm{c}} z \chi \eta_{0} \frac{1}{\alpha} .
$$

La durée de ce régime transitoire varie donc proportionnellement

- au volume du réservoir $S . z$,

- à la compressibilité du fluide $\chi$,

- à la viscosité du fluide $\eta_{0}$.

La viscosité de l'eau est approximativement de une centipoise, alors que celle d'un polymère fondu est de une à cent millions de fois plus élevée $\left(10^{4}\right.$ à $\left.10^{6} \mathrm{Po}\right)$. La compressibilité de l'eau a une valeur approximative de $10^{-5} \mathrm{bar}^{-1}$ et celle d'un polymère $10^{-4} \mathrm{bar}^{-1}$. Comparativement aux fluides moléculaires, les fluides polymères à l'état fondu présentent un temps d'atteinte du régime stationnaire particulièrement grand. Le caractère non newtonien, c'est-à-dire la propriété qu'ont les polymères de voir leur viscosité décroître avec le cisaillement, ne fait que réduire partiellement ce temps. C'est bien la valeur exceptionnellement élevée de la viscosité des polymères fondus qui est à l'origine de la grande durée des régimes transitoires.

Le passage de l'équation (7) à l'équation (8) suppose que le coefficient $\chi$ soit indépendant de la pression, ce qui n'est en général pas réalisé.

Afin de tester la validité des calculs ci-dessus, nous avons utilisé l'équation (7) et relevé les valeurs de $\mathrm{d} P_{\mathrm{E}} / \mathrm{d} t$ pour différentes filières $(\alpha)$ et différents volumes de réservoir $(z)$. Nous calculons à chaque fois, à l'aide de l'équation (7), la valeur de la compressibilité $\chi$. La dispersion des résultats est de l'ordre de $20 \%$ :

$$
\chi=1,1 \pm 0,1 \times 10^{-4} \mathrm{bar}^{-1}
$$

Cette valeur numérique est en bon accord avec celles proposées pour des polymères semblables et mesurées par des méthodes plus classiques par Hellwege et Beret $[14,15]$.

1.4.4 Conclusions. - Si le caractère non monotone de la courbe d'écoulement est à l'origine de l'instabilité, la compressibilité du fluide polymère est une condition nécessaire à l'établissement d'un régime oscillatoire. A cause des très hautes viscosités des polymères fondus, le déphasage dans le temps entre le débit d'entrée et le débit de sortie est extrêmement grand. Il permet donc, le cas échéant, d'observer un régime oscillatoire dans un domaine de fréquences assez basses.

1.5 Les oscillations de RelaXation. - Une oscillation de relaxation est une oscillation auto-entretenue dont la forme de l'onde montre de rapides changements de pente ou de hauteur à certains points du cycle [8]. Le terme a été introduit pour la première fois par Van Der Pol [9] en 1926 pour décrire les phénomènes cycliques dans lesquels de l'énergie stockée graduellement se relaxe soudainement. Le nom de Van Der Pol est aussi attaché à une équation paramétrique décrivant ces phénomènes.

D'une manière générale $[10,11]$ on peut dire que :

- Ce sont des systèmes qui évoluent entre deux régimes de fonctionnement distincts et passent alternativement de l'un à l'autre. Au phénomène de décharge brusque due à un état de déséquilibre, consécutif à une charge lente, on donne le nom de relaxation.

- Il n'y a pas, contrairement à l'oscillateur harmonique, de position d'équilibre stable.

- Les amplitudes des oscillations sont bien déterminées et se conservent.

- La courbe caractéristique qui représente le débit, c'est-à-dire la dérivée par rapport au temps de la variable quantité, en fonction de la variable de tension possède une forme telle qu'à une même tension correspondent deux débits.

- Les courbes ont de brusques changements de courbures et même des points anguleux.

Les figures 2 et 3 sont en accord avec les énoncés ci-dessus. Mais l'identification définitive du caractère relaxatif a pu être menée à bien en tenant compte de la remarque que rapporte Soutif [12] à propos de l'influence de l'intensité du signal d'entrée.

Alors que la pulsation d'un oscillateur harmonique ne dépend que des caractéristiques de construction du système, l'amplitude de la vibration est directement liée à l'intensité de signal d'entrée.

Il en va tout autrement en ce qui concerne l'oscillateur de relaxation. L'amplitude du mouvement est une caractéristique propre du système alors que la fréquence d'oscillation dépend directement de l'intensité du signal d'entrée, comme l'illustre le vase de tantale (Fig. 5).

Il est clair, sur un tel exemple, que l'amplitude des oscillations de pression et de débit sont parfaitement définies par les dimensions géométriques de l'appareil ( $h_{1}, h_{2}$, diamètre et longueur du siphon et du tracé). $\mathrm{La}$ fréquence d'amorçage du siphon, donc des oscillations, est-elle directement liée à la vitesse avec laquelle le réservoir se remplit, c'est-à-dire au débit d'entrée $Q_{\mathrm{E}}$.

Nous avons observé que cette caractéristique de l'oscillateur de relaxation se retrouve dans le cas de notre système physique : le débit d'entrée $Q_{\mathrm{E}}$ influence 


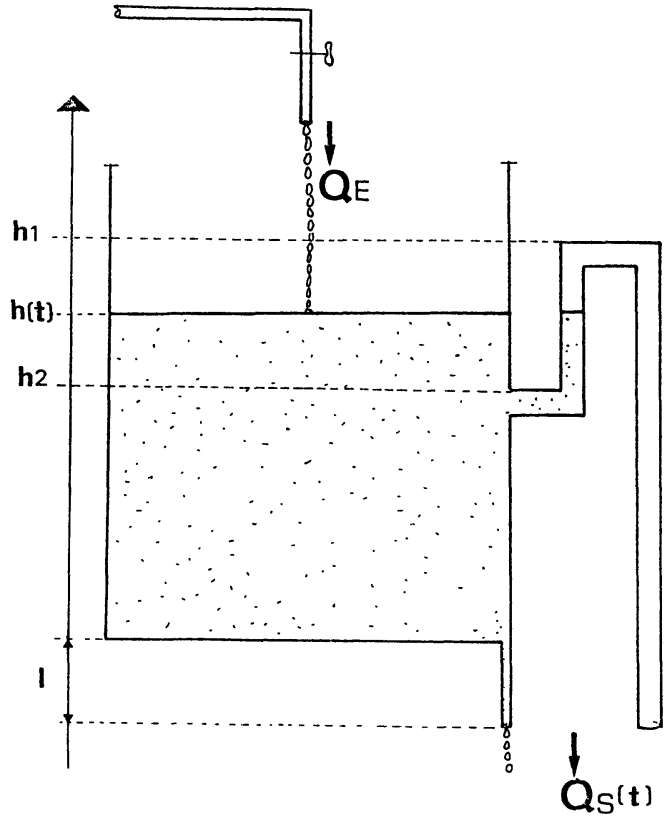

Fig. 5. - Vase de tantale : pour un certain domaine de débit d'entrée la hauteur d'eau et le débit à la sortie sont deux fonctions périodiques du temps. Les oscillations disparaissent pour des valeurs supérieures et inférieures du débit d'entrée.

[Tantalus's pot : for a definite domain of flow rate at the tap, $Q_{\mathrm{E}}$ the height of the water level and the exit flow rate, $Q_{s}$, are two periodical functions of time. For lower and higher values of $Q_{\mathrm{E}}$ oscillations disappear.]

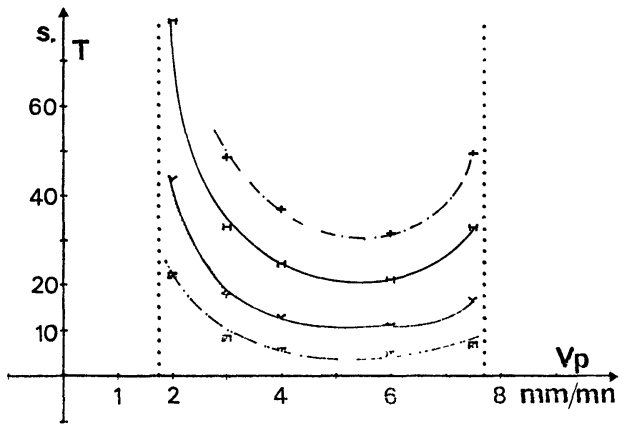

Fig. 6. - Influence de la vitesse du piston (ou du débit d'entrée) sur la période d'oscillation $T$ du système.

[Influence of the piston speed (or of the entry flow rate) on the oscillation period of the system, $T$.]

la période d'oscillation (Fig. 6) mais pas son amplitude.

En conclusion, il s'avère que l'on peut classer le phénomène d'instabilité d'écoulement capillaire des polyéthylènes de haute densité fondus, dans la famille des oscillateurs de relaxation.

Toutes les observations faites jusqu'à présent concourrent effectivement à cette conclusion. Similitude de comportement avec le vase de tantale, amplitude d'oscillation déterminée, courbes anguleuses, existence de deux courbes $P(Q)$, etc...

Dans une deuxième partie, nous nous attacherons à mettre en évidence la partie capacitive et résistive du système et nous ferons une analyse quantitative de la période des oscillations.

2. Etude quantitative. - Les oscillateurs de relaxation sont constitués d'un élément capable de stocker de l'énergie, dénommé élément capacitif, et d'un élément susceptible de dissiper cette énergie, ou élément résistif. Il en est ainsi du vase de tantale : la capacité est le récipient lui-même et la résistance celle des conduits d'écoulement. Quels sont, dans le cas du PEhd fondu, dans un rhéomètre capillaire, les éléments résistifs et capacitifs.

L'énergie stockée est l'énergie élastique emmagasinée dans le système physique. Elle est fonction du volume de matière comprimée, de la pression et de la compressibilité du polymère fondu. L'énergie dissipée est celle due à l'écoulement visqueux dans le système physique. Comme la quasi-totalité du volume du système est située dans le canal d'essai et comme sa résistance à l'écoulement est très faible par rapport à celle de la filière, nous disons que le canal d'essai est l'élément capacitif du système. De même puisque toute la résistance à l'écoulement est concentrée dans la filière nous disons que celle-ci est l'élément résistif.

2.1 L'éLÉMENT CAPACITIF. - Le contenu du canal d'essai du rhéomètre (Fig. 1) peut être considéré à chaque instant comme un corps élastique à une dimension de dureté $k$.

$$
k=-\frac{\mathrm{d} F}{\mathrm{~d} z}=\frac{S_{\mathrm{c}}}{\chi \cdot z} .
$$

La dureté $k$ du ressort équivalent est proportionnelle à la section $S_{\mathrm{c}}$ du canal et inversement proportionnelle à la longueur utile $z$ du canal et à la compressibilité $\chi$ du fluide considéré. Puisque $z$ est une fonction du temps, $k$ l'est aussi. La dureté du ressort tend vers l'infini quand le piston s'approche de plus en plus de la filière.

2.2 L'ÉLÉMENT RÉSISTIF. - Dans le repère du laboratoire le fluide s'écoule à l'intérieur de la filière immobile. Celle-ci est donc soumise à un ensemble de forces de réaction dont la résultante compense les forces d'entraînement dues à l'action du fluide. Dans le cas de l'écoulement, nous convenons de définir la résistance $R_{\mathrm{f}}$ du conduit selon :

$$
R_{\mathrm{f}}:=\frac{\mathrm{d} P}{\mathrm{~d} Q}
$$

2.2.1 Loi linéaire. - Dans le cas particulier des fluides newtoniens, la différence de pression d'une extrémité à l'autre du conduit varie linéairement avec le débit volumique selon la loi de Poiseuille

$$
P=\frac{8 \ln _{0}}{\pi R^{4}} Q
$$


La résistance que le conduit oppose à un tel fluide ne dépend donc pas du débit, mais uniquement de la viscosité du fluide $\eta_{0}$ et des dimensions géométriques de la filière, $l$ et $R$.

2.2.2 Loi non linéaire. - a) Ecoulement capillaire d'un fluide visqueux. - Soit un fluide visqueux incompressible. Tant qu'il est laminaire l'écoulement de ce fluide est de symétrie cylindrique. En écoulement permanent la vitesse $V$ d'une particule ne dépend que de sa coordonnée radiale $r$.

$$
\left.\frac{\partial V}{\partial z}\right]_{r}=0
$$

La valeur de la contrainte de cisaillement $s$ qui règne au point de rayon $r$ lorsque le régime est permanent, est directement proportionnelle au gradient axial de pression qui règne dans le tube :

$$
s(r)=\frac{r}{2} \frac{\partial P}{\partial z} \simeq \frac{r}{2} \frac{P_{\mathrm{E}}}{l} .
$$

Soit deux cylindres concentriques voisins, situés à l'abscisse $v$ et $v+\mathrm{d} v$. L'analyse montre que ce type de déformation est un cisaillement simple pour lequel la vitesse de cisaillement $\gamma(r)$ est définie par :

$$
\dot{\gamma}=\left|\frac{\mathrm{d} v}{\mathrm{~d} r}\right| \text {. }
$$

Si un fluide s'écoule dans un tube de rayon $R$ avec un débit volumique $Q$, il subit une perte de charge $P$. La vitesse de cisaillement à la paroi du tube $\gamma_{\mathrm{p}}$ 'exprime alors selon l'équation (12) de Rabinowitsch :

$$
\dot{\gamma}_{\mathrm{p}}=\frac{4 Q}{\pi R^{3}} \frac{3 n+1}{4 n}
$$

avec $n(Q)$ l'indice d'écoulement défini selon

$$
n(Q)=\frac{\mathrm{d} \lg P}{\mathrm{~d} \lg Q} .
$$

b) Résistance d'écoulement d'une filière capillaire. - Soit un polymère fondu s'écoulant dans une filière capillaire. En ne considérant que son comportement visqueux, son écoulement est décrit par les équations (11) et (12).

$\mathrm{La}$ résistance de la filière à l'êcoulement s'écrit donc :

$$
R_{\mathrm{f}}=\frac{\mathrm{d} P}{\mathrm{~d} Q}=\frac{2 l}{\pi R^{4}}\left(\frac{3 n+1}{n}\right) \frac{\mathrm{d} s(R)}{\mathrm{d} \dot{\gamma}_{\mathrm{p}}} .
$$

En introduisant l'expression de la viscosité apparente,

$$
\eta:=\frac{s(R)}{\dot{\gamma}_{\mathrm{p}}}
$$

il vient l'expression finale

$$
R_{\mathrm{f}}=\frac{2 l}{\pi R^{4}}(3 n+1) \eta(\dot{\gamma})
$$

La résistance d'une filière à l'écoulement d'un polymère fondu dépend des dimensions géométriques de la filière, de la viscosité apparente et de l'indice d'écoulement du fluide.

c) Exemple : courbe d'écoulement. - Comme illustration aux calculs ci-dessus nous traçons dans la figure 7 , la courbe $\eta(\dot{\gamma})$ obtenue pour un PEhd à $190^{\circ} \mathrm{C}$. Le comportement pseudoplastique du polymère se traduit par une décroissance continue de la

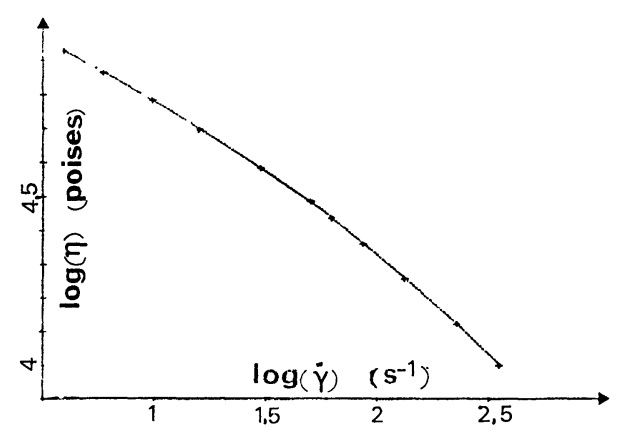

Fig. 7. - Variation de la viscosité en cisaillement simple du polyéthylène à $190^{\circ} \mathrm{C}$ en fonction de la vitesse de cisaillement $\dot{\gamma}$.

[Variation of the simple shear viscosity of polyethylene at $190{ }^{\circ} \mathrm{C}$ in function of the shear rate, $\dot{\gamma}$.]

viscosité $\eta$. L'indice d'écoulement $n$ est relié à la pente de la courbe par

$$
\frac{\mathrm{d} \lg \eta}{\mathrm{d} \lg \dot{\gamma}}=n-1 .
$$

Il décroît avec la vitesse de cisaillement. La résistance $R_{\mathrm{f}}$ est donc une fonction décroissante du débit dans la gamme de cisaillement étudiée.

d) Mesure de la période d'oscillation. - On sait que la période d'oscillation d'un système relaxatif est proportionnelle à la valeur de sa partie capacitive.

Nous avons mesuré la période $T$ de l'écoulement pour différents volumes de réservoirs et pour différentes filières. Un exemple de résultats est consigné dans la figure 8 . Il y apparaît que la période $T$ varie linéairement avec le volume du réservoir, ce qui confirme nos conclusions quant à la nature des oscillations.

Seule l'existence d'une ordonnée positive non nulle $T_{0}$ est peut-être inattendue.

Mais il convient de rappeler l'hypothèse émise au début de ce paragraphe concernant la répartition spatiale des éléments résistifs et capacitifs. Or, lorsque le volume du réservoir tend vers zéro, il devient incorrect de négliger le volume propre de la filière par rapport à celui du réservoir. $\mathrm{La}$ fonction capaci- 


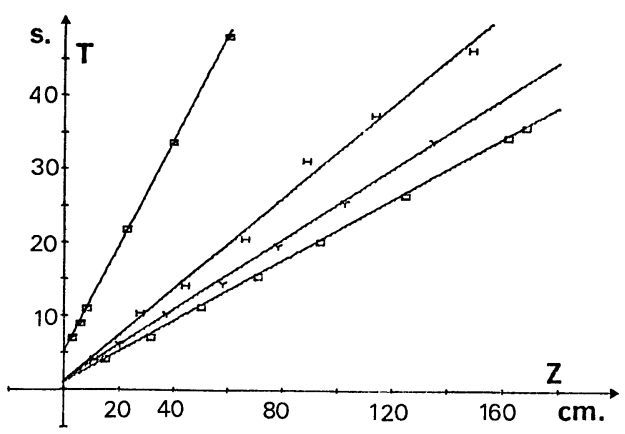

Fig. 8. - Influence du volume du réservoir sur la période d'oscillation du système. La variable $z$ mesure la distance entre le canal et la filière. La vitesse du piston est $(\mathrm{mm} / \mathrm{mm}): \square 2, \mathrm{H} \mathrm{7,5,} \mathrm{Y} \mathrm{4,} \square 6$.

[Influence of the volume of the reservoir on the period of the oscillation. The variable, $z$, measures the distance between the die and the piston. The piston speed is (mm/mn) : $\square 2, \mathrm{H} \mathrm{7,5,Y} \mathrm{4, \square 6.]}$

tive n'est plus localisée en amont de la fonction résistive mais tend à se superposer avec celle-ci. Le modèle de l'oscillateur linéaire ne convient donc plus.

Et, pour être répartie uniformément dans toute la filière à réservoir nul, la fonction capacitive n'en est pas pour autant nulle elle-même.

Il en résulte que la période d'oscillation tend vers une valeur finie non nulle $T_{0}$ que nous appelons période d'auto-oscillation de la filière sur elle-même.

L'interprétation physique de la valeur de $T_{0}$ que nous venons d'émettre est supportée par les deux résultats expérimentaux suivants :

1) Pour une même filière, la valeur de $T_{0}$ ne dépend pas du diamètre du canal réservoir. Ceci montre bien que la période d'auto-oscillation ne dépend que de la filière et non pas du système d'alimentation.

2) La période d'une oscillation de relaxation étant

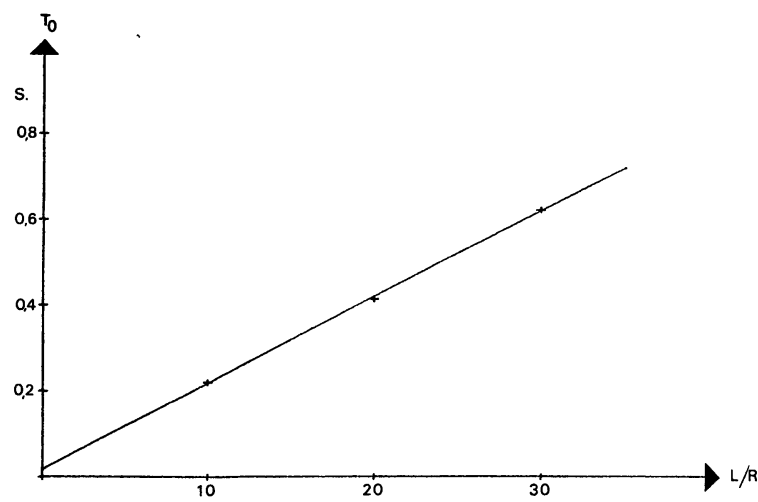

Fig. 9. - Variation de la période d'auto-oscillation $T_{0}$ (= limite de $T$ pour $z \rightarrow 0$ ) avec la longueur de la filière (à rayon constant).

[Variation of the self-oscillation period, $T_{0}\left(T_{0}=\right.$ limite $T$ for $z \rightarrow 0$ ) with the length of the die (at constant radii).]

proportionnelle à la valeur de la capacité, nous avons mesuré la valeur de $T_{0}$ pour trois filières de même rayon et de longueur différente. Dans le cas de l'autooscillation, la capacité est délocalisée dans tout le volume de la filière. Nous trouvons bien (Fig. 9) que $T_{0}$ est directement proportionnelle au volume de la filière [13].

3. Conclusion. - L'écoulement capillaire du polyéthylène de haute densité à l'état fondu donne lieu à des oscillations de relaxation. La nature résistive du système est liée à la viscosité du polymère, la nature capacitive à sa compressibilité hydrostatique.

A cause de la très grande valeur de la viscosité des polymères fondus, la fréquence des oscillations est assez basse et la période, aisément mesurable, est de l'ordre de grandeur de la dizaine de seconde.

\section{Bibliographie}

[1] WeILl, A., Thèse Strasbourg (1978).

[2] Petrie, C. J. S. et DenN, M. M., AIChEJ. 22-2 (1976) 209.

[3] Dennisson, M. T., Trans. J. Plast. Inst. 803 (1967).

[4] Den Otter, J. L., Rhéol. Act. 10 (1971) 200.

[5] Lupton, J. M. and Register, J. W., Pol. Eng. Sci. 235 (1965).

[6] Bergem, N., Proc. VII ${ }^{\text {th }}$ Int. Cong. Rheol., Chalmers Un. Techn. Gothenburg, 50 (1976).

[7] Weill, A., Guerdoux, L., Druz, J., Ann. Techn. Conf. preprints, SPE, New-Orleans (1979)

[8] Tanner, R. I., Encyclopedic Dictionnary of Physics (Pergamon Press) 1962.
[9] VAn der Pol, B., Phil. Mag. $7^{\text {th }}$ series, vol. 2 (1926).

[10] Andronow, A. A. and Chaikin, C. E., Theory of oscillations (English Translation) (Princetone : The University Press).

[11] MinORSKY, N., Introduction to non linear mechanics, Ann Arbor (Edwards).

[12] Soutif, M., "Vibrations, propagation, diffusion " (Dunod, Paris) 1970.

[13] Bouilloux, A., rapport D.E.A., Strasbourg (1979).

[14] Hellwege, K. H. and Al., Koll. ZEIT. Pol. 183-2 (1962) 110.

[15] Beret, S., J. Appl. Pol. Sci. 18 (1974) 3779.

[16] Huseby, T. W., Trans. Soc. Rhéol. $10: 1$ (1966) 181. 